\title{
YET ANOTHER CHARACTERIZATION \\ OF THE SINE FUNCTION
}

\section{ROBERT GERVAIS}

Département de Mathématiques Collège milltalre royal de Saint-Jean

SaInt-Jean, Québec, Canada J0J 1RO
LEE A. RUBEL

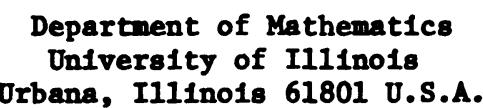

(Recelved February 14, 1980)

ABSTRACT. In this expository paper, it 18 shown that if an entire function of exponential type vanishes at least once in the complex plane and if it has exactly the same number of zeros (counting multiplicities) as its second derivative, then this function must take the form $\operatorname{Asin}(B z+C)$. KEY WORDS AND PHRASES. Entire function of exponential type, Jensen's formula, Llonville's theorem, Polsson Integral formula.

1980 MATHEMATICS SUBJECT CLASSIFICATION CODES: 30C15, 30D15, 30D35, $33 A 10$.

We give here a characterization of the sine function, and present a proof that uses several of the standard results of the elementary theory of functions of one complex variable. We make no claim to depth or orfginality of method. Our Intention is mostly expository - to provide an illustration of the elementary theory in action. We have taken pains to keep the exposition elementary and complete. Since more advanced methods (see, e.g. Wittich [8]) can be used to 
get stronger results in much less space, the reader could consider this article as an Invitation to the use of Nevanlinna theory in the study of differential equations.

In the set of entire functions, it is customary to classify functions according to the growth of their modulus. In this spirit, we give the following definftion: an entire function $f$ is of exponential type if there exist two real positive constants $C$ and $\tau$ such that

$$
|f(z)| \leq C e^{\tau|z|} \text { for all } z \in \emptyset
$$

where designates the complex plane.

If $f$ is a function of exponential type, then for every $z \in \notin$ such that $|z|=r>0$, we may write

$$
f^{\prime}(z)=\frac{1}{2 \pi 1} \int_{|\zeta-z|=1} \frac{f(\zeta)}{(\zeta-z)^{2}} d \zeta
$$

In order to get the estimate

$$
\begin{aligned}
\left|f^{\prime}(z)\right| & \leq \underset{|\zeta| \leq x+1}{\max |f(\zeta)|} \\
& \leq c e^{\tau(x+1)}=C^{\prime} \cdot e^{\pi r} .
\end{aligned}
$$

The last inequality is obtained via the preceding definition of a function of exponential type, and we may deduce from this inequality that the derivative of a function of exponential type is itself a function of exponential type.

The theorem we are about to establish may be formulated in the following way:

THEOREM A. Let $f$ be an entire function of exponential type, possessing at least one zero. If $f$ is such that $z$ is a zero of multiplicity $m$ of $f$ if and only If $z$ is also a zero of multiplicity $m$ of $f^{\prime \prime}$, the second derivative of $f$, then $f$ necessarily has the form

$$
f(z)=A \sin (B z+C)
$$

where $A, B$ and $C$ are three complex constants. 
To facilitate the exposition, we introduce the class $S$ of entire functions $f$ of exponential type that have at least one zero in the complex plane and that have the following property: $z$ is a zero of $f$ if and only if $z$ is a zero of $f$ ", counting multiplicities. For convenience, we shall eliminate the function constantly 0 from $S$. The functions

$$
\sin (z)=\frac{e^{1 z}-e^{-1 z}}{21} \text { and } \cos (z)=\frac{e^{1 z}+e^{-1 z}}{2}
$$

are examples of members of $S$. More generally, $f(z)=A \sin (B z+C)$ is a function In $S$, and the preceding theorem asserts that every element of $S$ is of this form.

We turn now to the proof of theorem $A$.

Let $f$ be a function in $S$. Then the function

$$
\phi(z)=\frac{f^{\prime \prime}(z)}{f(z)}
$$

Is an entire function without zeros, and we shall show that, In this case, it must take the form $\phi(z)=e^{h(z)}$ for some entire function $h$. We observe that $\phi^{\prime} / \phi$ is 1tself an entire function that must be the derivative of an entire function $\psi, 1 . e . \psi^{\prime}=\phi^{\prime} / \phi$. Consider now the new function

$$
H(z)=\phi(z) e^{-\psi(z)} \text {. }
$$

If we calculate the derivative of $H$, we get

$$
\begin{aligned}
H^{\prime}(z) & =e^{-\psi(z)}\left\{\phi^{\prime}(z)-\phi(z) \psi^{\prime}(z)\right\} \\
& =e^{-\psi(z)}\left\{\phi^{\prime}(z)-\phi(z) \frac{\phi^{\prime}(z)}{\phi(z)}\right\} \\
& =0,
\end{aligned}
$$

and we may conclude that

$$
H(z)=\phi(z) e^{-\psi(z)}=C .
$$

Here, $C$ is a constant. Hence each element $f \in S$ satisfles the differential equation

$$
f^{\prime \prime}(z)=f(z) e^{h(z)}
$$


for some entire function $h$.

We show now that in fact the function $h$ must be a polynomial of degree at most one. To do this, we shall use Jensen's formula (cf. Conway [1], p. 283):

$$
\log |f(0)|=\frac{1}{2 \pi} \int_{0}^{2 \pi} \log \left|f\left(r e^{1 \theta}\right)\right| d \theta-\sum_{k=1}^{n} \log \left(\frac{r}{\left|g_{k}\right|}\right) .
$$

Here it is supposed that $f$ is holomorphic in $|z| \leq r$, and that $a_{1}, \ldots, a_{n}$ are the zeros of $f$ contained in $|z|<r$, repeated as many times as their multiplicity indicates. From this inequality, we deduce that

$$
\log |f(0)| \leq \frac{1}{2 \pi} \int_{0}^{2 \pi} \log \left|f\left(r e^{1 \theta}\right)\right| d \theta \text {. }
$$

With the notation

$$
\log ^{+} t=\left\{\begin{array}{ll}
\log t & \text { for } t \geq 1 \\
0 & \text { for } 0<t<1
\end{array} \text { and } \log ^{-} t= \begin{cases}0 & \text { for } t>1 \\
-108 t & \text { for } 0<t \leq 1\end{cases}\right.
$$

we may write

$$
\log t=\log ^{+} t-\log ^{-} t .
$$

Thus, if $f 18$ in $S$, and if we moreover suppose that $|f(0)| \geq 1$, then we have

$$
\begin{aligned}
0 \leq \log |f(0)| \leq & \frac{1}{2 \pi} \int_{0}^{2 \pi} \log \left|f\left(r e^{1 \theta}\right)\right| d \theta \\
& =\frac{1}{2 \pi} \int_{0}^{2 \pi} \log ^{+}\left|f\left(r e^{1 \theta}\right)\right| d \theta- \\
& \frac{1}{2 \pi} \int_{0}^{2 \pi} \log \left|f\left(r e^{1 \theta}\right)\right| d \theta
\end{aligned}
$$

and we thus obtain 


$$
\begin{aligned}
\int_{0}^{2 \pi} \log \left|f\left(r \mathrm{~g}^{-1 \theta}\right)\right| \mathrm{d} \theta & \leq \int_{0}^{2 \pi} 10 g^{+}\left|f\left(r \mathrm{e}^{1 \theta}\right)\right| d \theta \\
& \leq \int_{0}^{2 \pi} 10 g^{+}\left(\mathrm{Ce}^{\tau r}\right) d \theta \\
& \leq \mathrm{C}_{1} \mathrm{x}
\end{aligned}
$$

for a constant $C_{1}$. Finally, using the triangle inequality, we deduce the result

$$
\begin{aligned}
\int_{0}^{2 \pi}|\log | f\left(r e^{1 \theta}\right)|| d \theta & \leq \int_{0}^{2 \pi} \log \left|f\left(r e^{1 \theta}\right)\right| d \theta+\int_{0}^{2 \pi} \log ^{+}\left|f\left(r e^{1 \theta}\right)\right| d \theta \\
& \leq 2 C_{1} r .
\end{aligned}
$$

In the same fashion, we could demonstrate that, for another constant $\mathrm{C}_{2}$,

$$
\int_{0}^{2 \pi}|\log | f^{\prime \prime}\left(r e^{1 \theta}\right)|| d \theta \leq 2 c_{2} r
$$

on supposing also that $\left|f^{\prime \prime}(0)\right| \geq 1$.

Returning to the function $h$ of the identity (2) and writing

$$
h(z)=u(z)+1 v(z)
$$

we may use equation (2) to write

$$
\log \left|f^{\prime \prime}\left(r e^{1 \theta}\right)\right|=\log \left|f\left(r e^{1 \theta}\right)\right|+u\left(r e^{1 \theta}\right)
$$

This leads us (taking account of the preceding inequalities) to the inequality

$$
\begin{aligned}
\int_{0}^{2 \pi}\left|u\left(r e^{1 \theta}\right)\right| d \theta & \leq \int_{0}^{2 \pi}|\log | f\left(r e^{1 \theta}\right)|| d \theta+\int_{0}^{2 \pi}|\log | f^{\prime \prime}\left(r e^{1 \theta}\right)|| d \theta \\
& \leq C_{3} r
\end{aligned}
$$

for a constant $\mathrm{C}_{3}$. Finally, we write the representation of $\mathrm{h}$ as a complex Polsson 
Integral (cf. Rudin [7], p.228):

$$
h\left(r e^{1 \phi}\right)=\frac{1}{2 \pi} \int_{0}^{2 \pi}\left\{\frac{2 r e^{1 \theta}+r e^{1 \phi}}{2 r e^{1 \theta}-r e^{1 \phi}}\right\} u\left(2 r e^{1 \theta}\right) d \theta
$$

and use the last inequality to obtain

$$
\begin{aligned}
& \left|\mathrm{h}\left(r \mathrm{e}^{1 \phi}\right)\right| \leq \frac{1}{2 \pi}\left\{\int_{0}^{2 \pi}\left|\mathrm{u}\left(2 \mathrm{re} \mathrm{r}^{1 \theta}\right)\right| \mathrm{d \theta}\right\} \underset{\substack{0 \leq \theta \leq 2 \pi \\
0 \leq \phi \leq 2 \pi}}{\max }\left|\frac{2 r \mathrm{e}^{1 \theta}+r \mathrm{re}^{1 \theta}-r \mathrm{e}^{1 \phi}}{2 \phi}\right| \\
& \leq \frac{C_{3} D}{2 \pi} r
\end{aligned}
$$

where the constant $D$, Independent of $r$, satisfies the inequality

$$
\max _{\substack{0 \leq \theta \leq 2 \pi \\ 0 \leq \phi \leq 2 \pi}}\left|\frac{2 r e^{1 \theta}+r e^{1 \phi}}{2 r e^{1 \theta}-r e^{1 \phi}}\right| \leq D .
$$

Since $h$ is an entire function that grows no faster than a constant multiple of the Independent variable, we may use a direct consequence of Liouville's theorem to conclude that $h$ is a polynomial of degree at most 1 .

Thus, if we summarize the present situation, we have, for every $f \in S$ such that

$$
|f(0)| \geq 1 \text { and }\left|f^{\prime \prime}(0)\right| \geq 1
$$

the Identity

$$
f^{\prime \prime}(z)=f(z) e^{A z+B}
$$

or equivalentiy

$$
f^{\prime \prime}(z)=C e^{A z} f(z)
$$

for two possibly complex constants $A$ and $C$. We now show directly that the two hypotheses in (3) only constitute a simple normalization. In the first place, if we had $f(0)=0$ (and hence $f^{\prime \prime}(0)=0$ since $f \in S$ ), the trouble would be that $|f(0)|<1$ or $\left|f^{\prime \prime}(0)\right|<1$, so we could take $f_{1}(z)=\alpha f(z)$ where 


$$
\alpha=\max \left\{\frac{1}{\mid f(0) T}, \frac{1}{\mid f^{\prime \prime}(0)}\right\} .
$$

The function $f_{1}$ belongs to $S$ and satisfies (3). If $f_{1}$ takes the form indicated in theorem $A$, then $f$ also does. In case $f(0)=0$ (and consequently $f^{\prime \prime}(0)=0$ ) then we perform the translation

$$
f_{2}(z)=f(z+\epsilon)
$$

where $\epsilon$ 18 a constant chosen so that $f_{2}(0) \neq 0$. Now one proceeds to show that $f_{2}$ has the required form, and hence that $f$ does.

In the sequel, we shall simplify the exposition by supposing, without loss of generality, that $f \in S$, and that $|f(0)| \geq 1$ and $\left|f^{\prime \prime}(0)\right| \geq 1$, so that $f$ satisfies (4).

Our aim, at this point, is to show that the constant A in (4) must be zero, so let us suppose otherwlse. For simplicity, we shall suppose $A=1$ in (4) since otherwlse we could consider the function

$$
F(z)=f\left(\frac{z}{A}\right)
$$

'which also belongs to $S$ and satisfies the differential equation

$$
F^{\prime \prime}(z)=C^{\prime} e^{z} F(z)
$$

where $C^{\prime}$ is a constant.

Let

$$
f(z)=\sum_{n=0}^{\infty} a_{n} z^{n}
$$

be the Taylor series of $f$. We may estimate the coefficlents as follows:

$$
\begin{aligned}
& \left|a_{n}\right|=\frac{\left|f^{(n)}(0)\right|}{n !}=\frac{1}{2 \pi 1} \int_{|\zeta|=r} \frac{f(\zeta)}{\zeta^{n+1}} d \zeta \\
& \leq \frac{1}{r^{n}} \max _{|\zeta|=r}|f(\zeta)| \\
& \leq \frac{C e^{\tau r}}{r^{n}} \text { for all } r>0 \text { and } n \geq 0 \text {. }
\end{aligned}
$$


Let us choose $r=n$ and use Stirilng's formula to deduce the estimate

$$
\begin{aligned}
\left|a_{n} n !\right|^{\frac{1}{n}} & \leq \frac{c^{\frac{1}{n}} e^{\tau}}{n}(n !)^{\frac{1}{n}} \\
& \leq \frac{c^{\frac{1}{n}} e^{\tau}}{n}(2 \pi n)^{\frac{1}{2 n}} \frac{n}{e}\left(1+\frac{1}{12 n-1}\right)^{\frac{1}{n}} \\
& \leq c^{\prime} \quad \text { for all } n \geq 0
\end{aligned}
$$

where we suppose the elementary fact that $\lim _{n \rightarrow \infty} n^{1 / n}=1$. Consequentiy we find

$$
\lim _{n \rightarrow \infty} \sup \left|a_{n} n !\right|^{\frac{1}{n}}=\rho<\infty,
$$

which signifies that the series

$$
\sum_{n=0}^{\infty} \frac{m^{n !}}{w^{n+1}}
$$

converges uniformly for $|w| \geq \rho^{\prime}>\rho$ and thus defines a function that is holomorphic in a neighborhood of infinity and that vanishes at $\infty$.

Now consider, for $\operatorname{Re}(w)>\max \{p, \tau\}$, the integral

$$
\begin{aligned}
& \phi(w)=\int_{0}^{\infty} f(t) e^{-w t} d t \\
& =\int_{0}^{\infty}\left\{\sum_{n=0}^{\infty} a_{n} t^{n}\right\} e^{-w t} d t \\
& =\sum_{n=0}^{\infty} a_{n} \int_{0}^{\infty} t^{n} e^{-w t} d t \\
& =\sum_{n=0}^{\infty} \frac{a_{n} n !}{w^{n+1}} .
\end{aligned}
$$

The interchange of the integration and the sumbation is justifled by (5) and Its consequences. By the remark of the preceding paragraph, the function $\phi$ thus defined 18 holomorphic in a right half-plane. On the other hand, we have remarked 
that the derivative of an entire function of exponential type 1s again of exponential type. We may apply this to do the following integration by parts

$$
\begin{aligned}
\phi(w) & =\int_{0}^{\infty} f(t) e^{-w t} d t \\
& =\frac{f(0)}{w}+\int_{0}^{\infty} f^{\prime}(t) \frac{e^{-w t}}{w} d t \\
& =\frac{f(0)}{w}+\frac{f^{\prime}(0)}{w^{2}}+\int_{0}^{\infty} f^{\prime \prime}(t) \frac{e^{-w t}}{w^{2}} d t .
\end{aligned}
$$

Finally, since $f^{\prime \prime}$ must satisfy the equation (4) with $A=1$, the function satisfles the following relation:

$$
w^{2} \phi(w)-w f(0)-f^{\prime}(0)=C_{\phi}(w-1)
$$

where $\mathrm{C}$ is the same constant as in (4). Now we have remarked above that since $\phi$ Is holomorphic in a right half-plane, (and recalling that $C \neq 0$ because we have ruled out $f \equiv 0$ ), the last inequality allows us to continue $\phi$ analytically to the whole complex plane, as follows. We know that $\phi 18$ holomorphic for $\operatorname{Re}(w) \geq B>$ $\max \{\rho, \tau\}$, and the preceding equation allows us to continue $\phi$ analytically to $\operatorname{Re}(w) \geq B-1$, then to $\operatorname{Re}(w) \geq B-2$, and so on, unt11 the whole complex plane 18 covered, moving to the left by a band of width 1 each time. But we know also that $\phi$ is holomorphic in a neighborhood of infinity. Hence the analytic continuation of $\phi$ is holomorphic on the whole Riemann sphere, and must therefore be a constant. This constant is actually zero, since $\phi(\infty)=0$. Now since we have

$$
f(z)=\sum_{n=0}^{\infty} a_{n} z^{n} \text { and } \phi(w)=\sum_{n=0}^{\infty} \frac{a_{n} n !}{w^{n+1}}
$$

where the coefficients $a_{n}, n=0,1,2, \ldots$, that appear in the two developments are the same, and since we have shown that $\phi \equiv 0$, we have $f \equiv 0$, which contradicts our exclusion of 0 from $S$. Hence the constant $A$ of equation (4) must be zero. 
All these considerations lead to the following situation: if $f \in S$ and if $|f(0)| \geq 1$ and $\left|f^{\prime \prime}(0)\right| \geq 1$ then $f$ must satisfy the differential equation

$$
f^{\prime \prime}(z)=C f(z)
$$

for a (possibly complex) constant $c \neq 0$. Write $c=|c| e^{1 \lambda}$ and consider the new function

$$
F(z)=f(\alpha z)
$$

where

$$
\alpha=\frac{1}{\sqrt{|c|}} e^{1\left(\frac{\pi-\lambda}{2}\right)} .
$$

This function $F$ also belongs to $S$ and satisfies the differential equation

$$
F^{\prime \prime}(z)=-F(z) \text {. }
$$

Now in the elementary theory of differential equations, it is shown that all solutions of this equation must be of the form

$$
F(z)=a e^{1 z}+b e^{-1 z}
$$

for two complex numbers $a$ and $b$. Since $F \in S$, it has at least one zero. This implies that $a \neq 0$ and $b \neq 0$. We may rewrite this equation in the form

$$
F(z)=c_{1} \cos z+c_{2} \sin z
$$

where $c_{1}=a+b$ and $c_{2}=1(a-b)$, and we remark that

$$
c_{1}^{2}+c_{2}^{2}=4 \mathrm{ab} \neq 0
$$

since $a \neq 0$ and $b \neq 0$. Let us choose $A=\sqrt{c_{1}^{2}+c_{2}^{2}}$ and $c=\operatorname{arc} \tan \frac{c_{2}}{c_{1}}$ to deduce from elementary trigonometry that

$$
F(z)=A \sin (z+C)
$$

and hence that

$$
f(z)=A \sin (B z+C)
$$

where A, B and C are three complex constants. Th1s concludes the proof of theorem A. 
Before ending, we remark that once it is established that a function $f$ of the class $S$ must satisfy a differential equation of the form (4), there are alternative elementary proofs at hand. The one we have chosen has the advantage of remaining in the field of functions of a complex variable, but one could alternatively proceed directly from the solution of (4) obtained by the classical methods of the theory of differential equations and a detailed examination of the solution to derive the conclusion of theorem $A$.

\section{REFERENCES}

1. J.B. Conway, Functions of One Complex Variable, Springer-Verlag (1973).

2. H. Delange, Caractérisations des fonctions circulaires, Bul1. Sc1. Math. (2) 91 (1967), 65-73.

3. R. Gervals and Q.I. Rahman, An extension of Carlson's theorem for ent1re functions of exponential type, Trans. Amer. Math. Soc. 235 (1978) $387-394$.

4. R. Gervais and Q.I. Rahman, An extension of Carlson's theorem for entire functions of exponential type II, J. Math. Anal. App. (2) 69 (1979) 585-602.

5. M. Ozawa, A characterization of the cosine function by the value distribution, Koda1 Math. J. 1 (1978) 213-218.

6. L.A. Rubel and C.C. Yang, Interpolation and unavoldable families of meromorphic functions, Michigan Math. J. 20 (1973) 289-296.

7. W. Rudin, Real and Complex Analysis, McGraw-H111 (1966).

8. H. Witt1ch, Neuere Untersuchungen Hber elndeutige analytische Funkt1onen, Springer-Verlag (1968). 


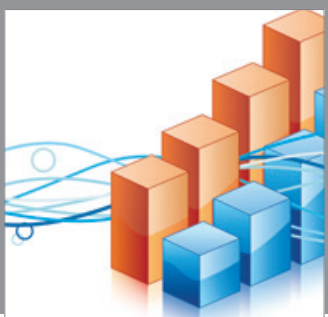

Advances in

Operations Research

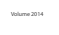

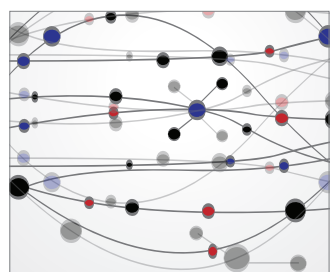

\section{The Scientific} World Journal
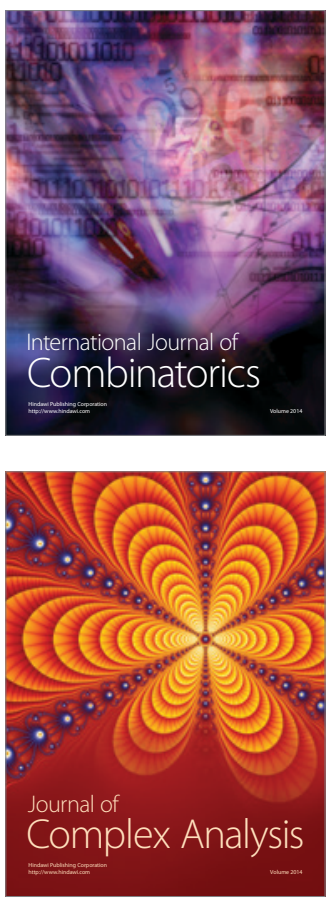

International Journal of

Mathematics and

Mathematical

Sciences
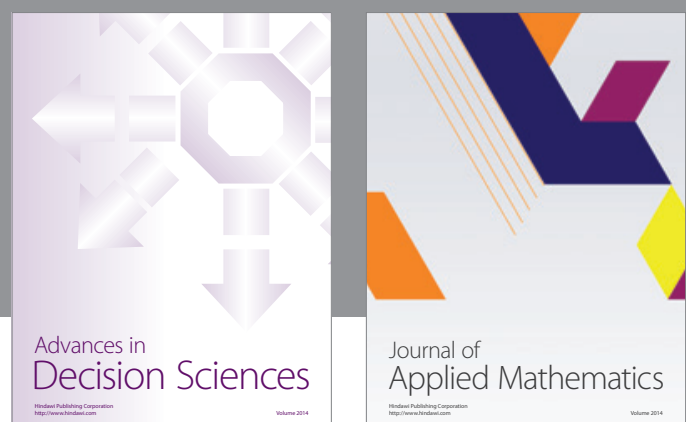

Journal of

Applied Mathematics
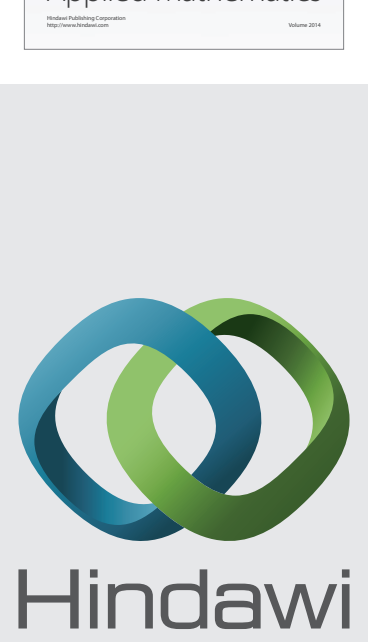

Submit your manuscripts at http://www.hindawi.com
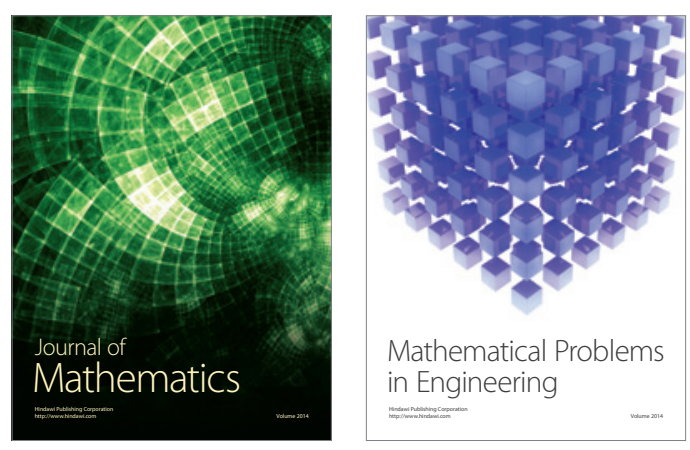

Mathematical Problems in Engineering
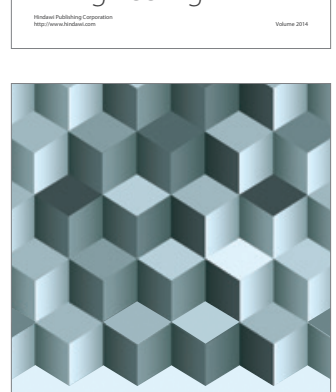

Journal of

Function Spaces
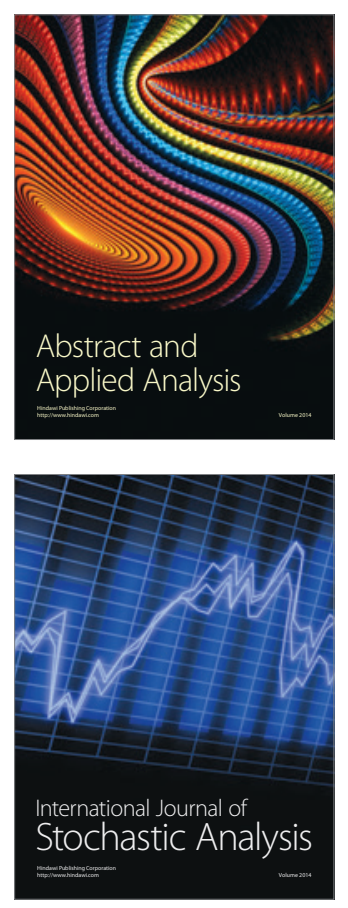

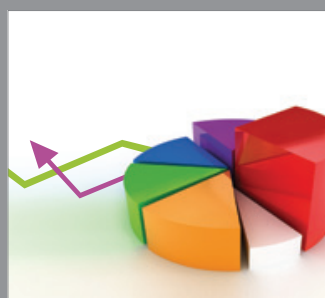

ournal of

Probability and Statistics

Promensencen
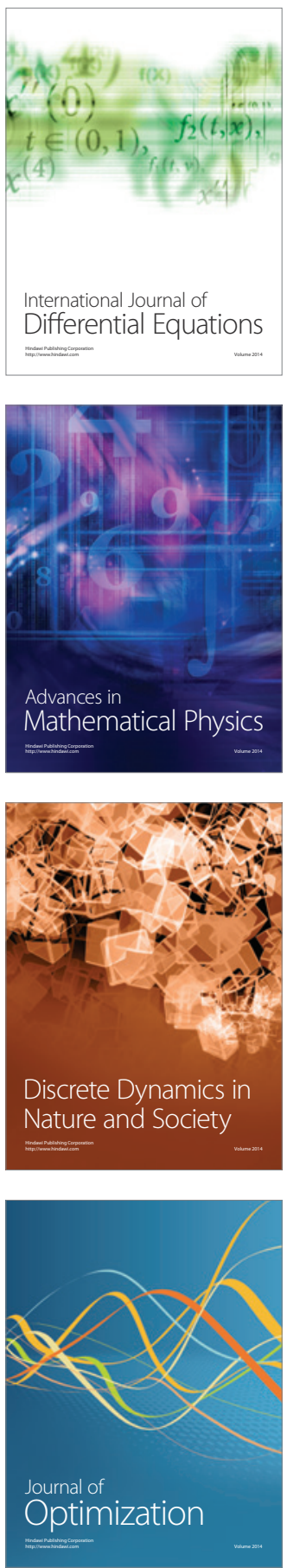